\title{
Cross-scale: multi-scale coupling in space plasmas
}

\author{
Steven J. Schwartz • Timothy Horbury • \\ Christopher Owen • Wolfgang Baumjohann • \\ Rumi Nakamura • Patrick Canu • Alain Roux • \\ Fouad Sahraoui · Philippe Louarn • \\ Jean-André Sauvaud • Jean-Louis Pinçon • \\ Andris Vaivads • Maria Federica Marcucci • \\ Anastasios Anastasiadis • Masaki Fujimoto • \\ Philippe Escoubet • Matt Taylor • \\ Steven Eckersley • Elie Allouis • \\ Marie-Claire Perkinson . \\ on behalf of the Cross-Scale Team
}

Received: 28 November 2007 / Accepted: 15 February 2008 / Published online: 15 March 2008

(C) Springer Science + Business Media B.V. 2008

\begin{abstract}
Most of the visible universe is in the highly ionised plasma state, and most of that plasma is collision-free. Three physical phenomena are responsible for nearly all of the processes that accelerate particles, transport material and energy, and mediate flows in systems as diverse as radio galaxy jets and supernovae explosions through to solar flares and planetary magnetospheres. These processes in turn result from the coupling amongst phenomena at
\end{abstract}

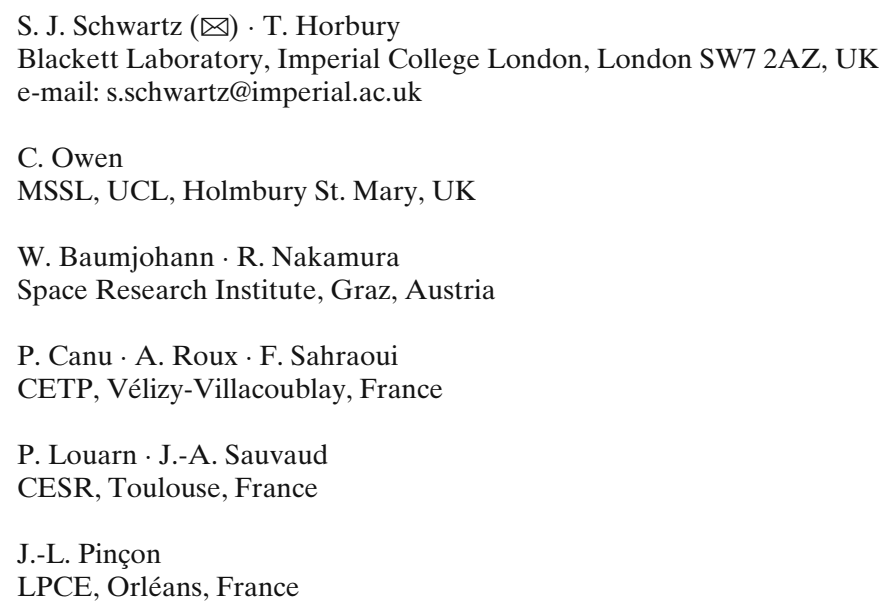

A. Vaivads

Swedish Institute of Space Physics, Uppsala, Sweden 
macroscopic fluid scales, smaller ion scales, and down to electron scales. CrossScale, in concert with its sister mission SCOPE (to be provided by the Japan Aerospace Exploration Agency-JAXA), is dedicated to quantifying that nonlinear, time-varying coupling via the simultaneous in-situ observations of space plasmas performed by a fleet of 12 spacecraft in near-Earth orbit. CrossScale has been selected for the Assessment Phase of Cosmic Vision by the European Space Agency.

Keywords Cross-scale • Cosmic Vision • Multi-scale coupling in plasmas • Space plasmas

PACS $90.05-\mathrm{a} \cdot 94.80 .+\mathrm{g} \cdot 95.40 .+\mathrm{s} \cdot 52.27 . \mathrm{h}$

\section{Scientific objectives}

Most of the visible universe is in the highly ionised plasma state, and most of that plasma is collision-free. Plasma processes are at work everywhere, from radio galaxy jets and supernovae explosions to solar flares, planetary magnetospheres, and terrestrial laboratories. Cross-Scale is a medium-sized ("M-class") mission dedicated to quantifying the coupling in plasmas between different physical scales. This cross-scale coupling, being highly variable and structured, controls the energisation and transport in most plasmas, many of which are difficult to observe.

As plasma regimes encounter each other, the absence of collisions raises fundamental questions about how energy is shared, and the physics controlled, amongst the three main elements (electrons, ions, and bulk flows). These constituents, each of which operates on its own physical scale, are coupled through electromagnetic fields.

M. F. Marcucci

IFSI, Rome, Italy

A. Anastasiadis

National Observatory of Athens,

Institute for Space Applications \& Remote Sensing, Athens, Greece

M. Fujimoto

ISAS, Kanagawa, Japan

P. Escoubet · M. Taylor

ESTEC, Noordwijk, The Netherlands

S. Eckersley $\cdot$ E. Allouis $\cdot$ M.-C. Perkinson

EADS Astrium UK Ltd., Stevenage, UK

on behalf of the Cross-Scale Team

URL: http://www.cross-scale.org

Springer 
Three fundamental physical processes operate to bring about the universal collisionless plasma coupling in physical environments where momentum and energy transfer is important.

Shock waves guide strong flows around obstacles or at interfaces between two flow regimes. They are important locations for the transfer of directed bulk flow energy into heat, with an attendant acceleration of energetic particles (see Fig. 1).

Magnetic reconnection releases stored magnetic energy to the plasma, and allows for exchange of material between previously isolated regions. Moreover, the consequent change in magnetic topologies provides a coupling between plasma regions which often drives the global scale dynamics of the system (see Fig. 2).

Turbulence transports energy from large scales at which it is input to small scales where it is dissipated. In the process, it interacts strongly, and often selectively, with plasma particle populations as either a source or sink (or both) of energy (see Fig. 3).

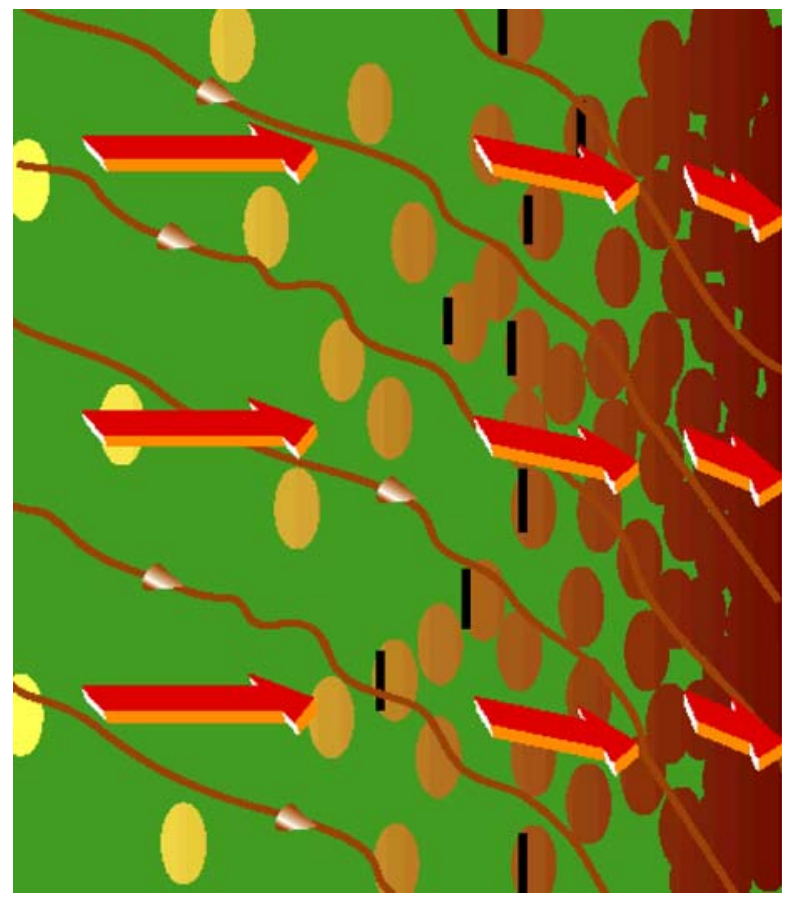

Fig. 1 Sketch of a collisionless shock under quasi-parallel conditions, in which the magnetic field threads the shock layer allowing the escape of particles into the oncoming flow. The resulting patchwork of field enhancements, shown as ovals, result in a configuration that is dramatically different to the traditional view of a shock as a 1-D planar transition from the incident, fast unshocked material on the left to the decelerated and "heated" plasma on the right. Such shocks are efficient accelerators of particles, and require simultaneous measurements at the largest fluid scales, intermediate patchwork scales, and smallest electron scales (at which there is locally steepening indicated by the black bars) [after 6] 


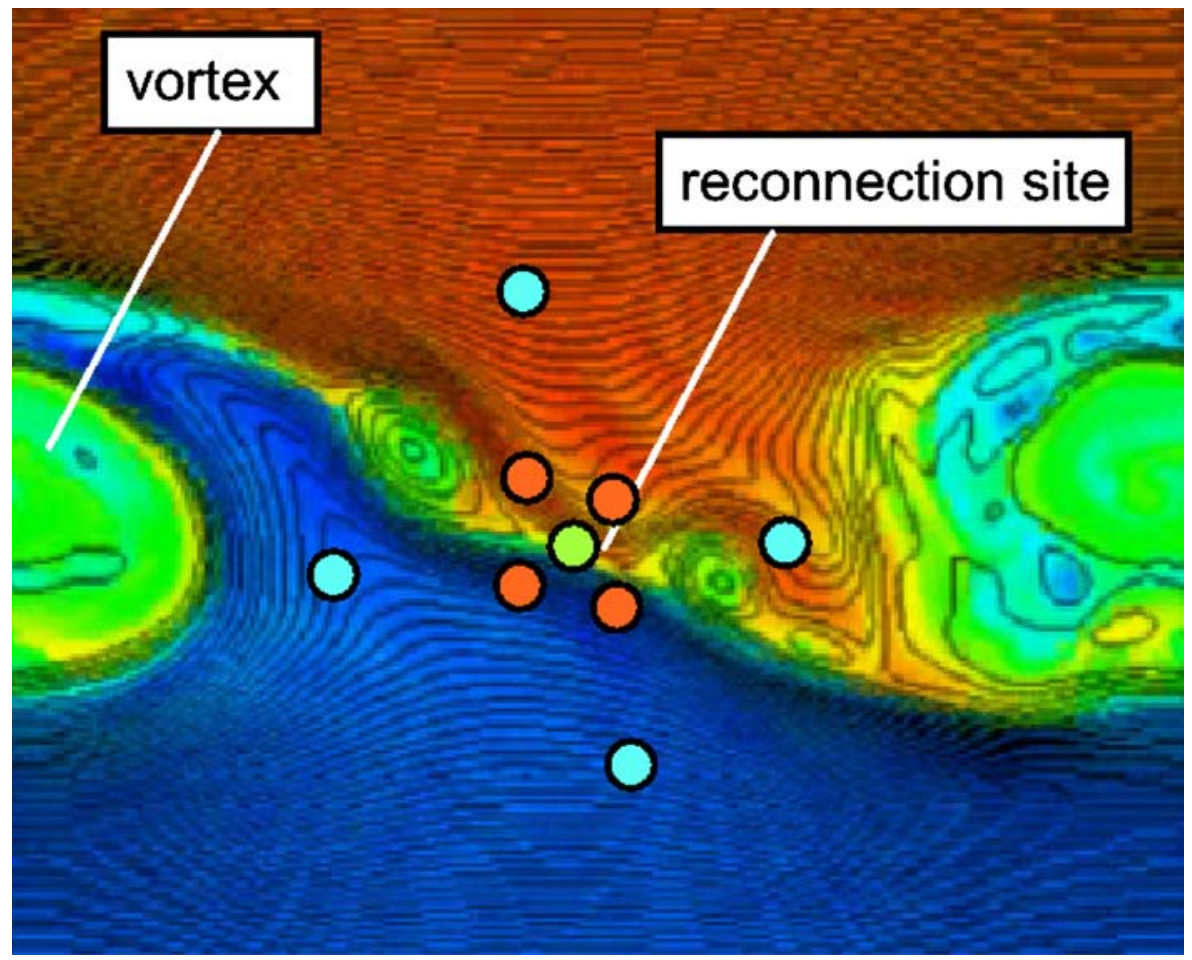

Fig. 2 Magnetic reconnection at a convoluted shear boundary, showing the deployment of nested observations necessary to unravel the dynamic coupling that changes the magnetic topology and accererates particles [see, e.g., 7]

Near-Earth space is a unique laboratory for quantifying the physics of these three processes. Breakthroughs have arisen due to the high quality of data that, unlike more distant regimes, is sampled directly by plasma and fields experiments on satellites.

To date, in situ measurements have focused on terrestrial phenomena, such as the mechanisms that populate the van Allen radiation belts. Dual spacecraft studies during the 1980 s began to address the real microphysics. Present generation missions (Cluster and MMS) utilise four spacecraft to sample a specific volume, and hence characterise the physics operating on the single scale corresponding to the spacecraft separation. By the time MMS has flown, we shall have a catalogue of behaviour that ranges from the smallest, electron scale, to the largest fluid-like phenomena.

That knowledge is incomplete due to the ambiguity and uncertainty about the dynamics and variability of the larger contextual scales (for the electron and ion scales) and of the internal micro-processes that mediate the energy exchange (for the larger scales). The complex, dynamic nonlinear coupling of scales and physical mechanisms can not be quantified without simultaneous information on all scales. 
Fig. 3 A computer simulation of 2D electron MHD turbulence, showing how plasma currents are spatially localised into thin sheets and whorls.

Many-point measurements, such as those from

Cross-Scale, are required to measure such structures in 3D in space plasmas over a range of scales not accessible in simulations [after 4]

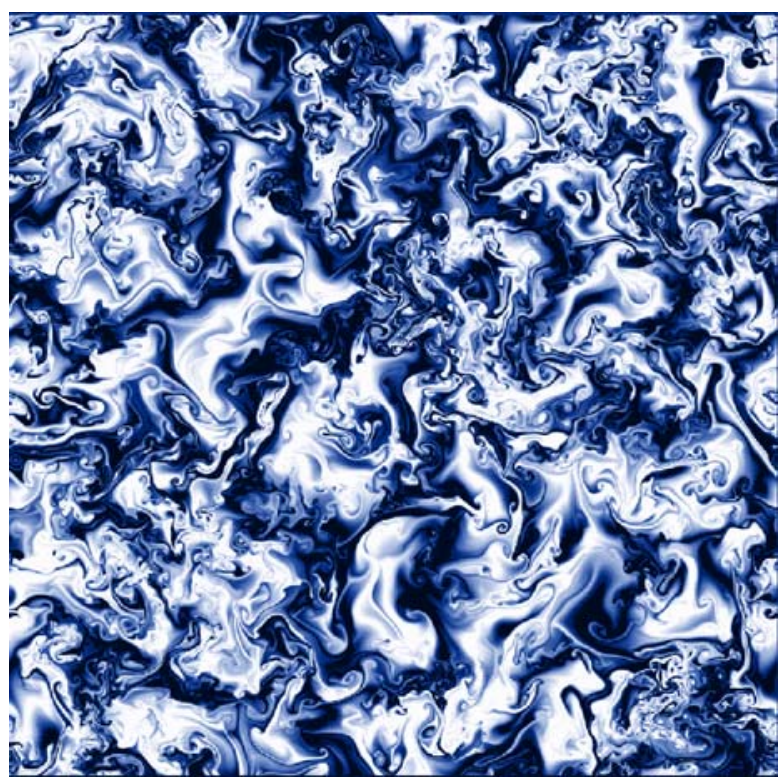

Further details of the Science Objectives can be found in the full proposal [9] and in earlier mission publications [e.g., 3, 5] and in the description of the Japanese SCOPE mission provided by Fujimoto et al. [2].

\section{Science questions}

Cross-Scale will target compelling and fundamental questions, such as:

- How do shocks accelerate and heat particles?

- How are particles accelerated in and around shocks?

- How is the energy incident on a shock partitioned?

- How do shock variability and reformation influence the acceleration process?

- How does reconnection convert magnetic energy?

- What initiates magnetic reconnection?

- How does the magnetic topology evolve?

- How does reconnection accelerate particles and heat plasma?

- How does turbulence control transport in plasmas?

- How does the turbulence cascade transfer energy across physical scales?

- How does the magnetic field break the symmetry of plasma turbulence?

- How does turbulence generate coherent structures? 
These address directly the Cosmic Vision question "How does the Solar System work?" by studying basic processes occurring "From the Sun to the edge of the Solar System." By quantifying the fundamental processes involved, the advances made by the mission will extend beyond the Solar System to plasmas elsewhere in the Universe.

\section{Mission strategy}

Cross-Scale will meets its scientific objectives by employing 3D concepts and techniques, pioneered by Cluster, simultaneously on three logarithmically spaced scales. Focused instrumentation on each scale then enables the dynamic coupling across those key scales to be measured.

\subsection{Mission profile}

Cross-Scale will employ 10 spacecraft which will fly with two highly complementary spacecraft from its sister mission SCOPE provided by JAXA. Together, they will form three nested tetrahedra, as shown in Fig. 4, to separate spatial and temporal variations simultaneously on the three key scales for the first time. In near-Earth space, the electron physics occurs on scales $\sim 2-100 \mathrm{~km}$, the ion physics on scales $\sim 50-2,000 \mathrm{~km}$, and the macroscopic fluid scales are typically $\sim 3,000-15,000 \mathrm{~km}$. Over the two year mission in a highly eccentric orbit the spacecraft will encounter various collisionless shocks, explore regions of both spontaneous and strongly driven reconnection, and investigate both nascent and highly evolved plasma turbulence.
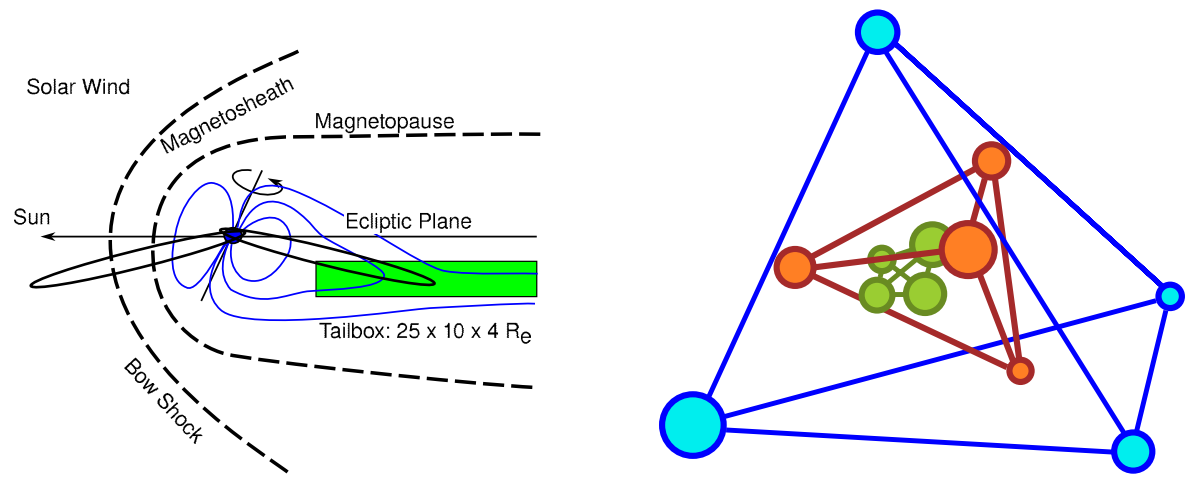

Fig. 4 The orbit of Cross-Scale (left) is designed to traverse the dayside solar wind, bow shock and magnetosheath, and 6 months later the nightside in the "tailbox" region. These provide examples under a variety of physical conditions of shock waves, magnetic reconnection, and turbulence. The nested configuration of spacecraft (right) are actually logarithmically spaced from $2-100 \mathrm{~km}$ at the smallest separations up to $15,000 \mathrm{~km}$ at the largest to measure simultaneously the 3 key scales: electron, ion, and fluid 


\subsection{Spacecraft}

The spacecraft will be identical in bus design (for economy) with modular bays that can accommodate instrument suites tailored to the relevant scale. The two JAXA SCOPE spacecraft complete the instrumentation and locations needed to meet the requirements. Dedicated hardware will determine the inter-satellite ranges at the smaller scales.

While some aspects of the spacecraft are challenging (X-band communications, large onboard memory, centralised processing, autonomous operation and data retrieval), there are no new technologies that must be established. The ten spacecraft will be dispensed by a vehicle with commercial heritage. Construction and calibration of the instruments is a large task (a factor $\sim 2$ larger than previous missions) but builds, together with the operation of a fleet of spacecraft, on recent European experience and success.

\subsection{Payload}

The payload consists almost entirely of proven technology, with heritage in recent missions (e.g., Cluster, Polar, Themis, STEREO) and well-advanced concepts (e.g., MMS). Although the spacecraft bus and subsystems are identi$\mathrm{cal}$, spacecraft devoted to different scales need different payload in terms of, e.g., time resolution, particle distributions vs. gross characteristics, and DC/AC fields. The largest demands come from the smallest scale (electron) spacecraft, with those at the fluid scale more modest.

To achieve this while benefiting from the economies of identical bus systems, the payload can be accommodated in universal mountings, such as modular bays or simple mounting plates. These plates present a common mechanical and electrical interface to the spacecraft, and are arranged on a single instrument shelf located at the top of the spacecraft. Figure 5 illustrates the concept, which provides ease of assembly, integration, and verification and facilitates replacement of faulty instruments. A thermal and electrical cover of some kind is needed, which could be a full or partial top to the entire spacecraft or specific to the individual bay.

Table 1 provides an overview of the instrument complement on each spacecraft, shown pictorially in Fig. 6. The main spacecraft resources are provided in Table 2.

The instrument complement on each spacecraft is targetted in terms of type of measurement, time resolution, and level of detail to match the highest science priorities (see [8] for details). The overall characteristics are shown in Table 3 with the deployment on the various spacecraft encapsulated in Table 1 and Fig. 6.

DC magnetometers (dual-sensors) will be deployed on all spacecraft, returning field vectors sampled at up to $\sim 100 \mathrm{~Hz}$ on the electron scale, and $1-10 \mathrm{~Hz}$ on the ion scale spacecraft. This data provides a robust roadmap of the 3D multi-scale morphology/connectivity, fundamental components of the 


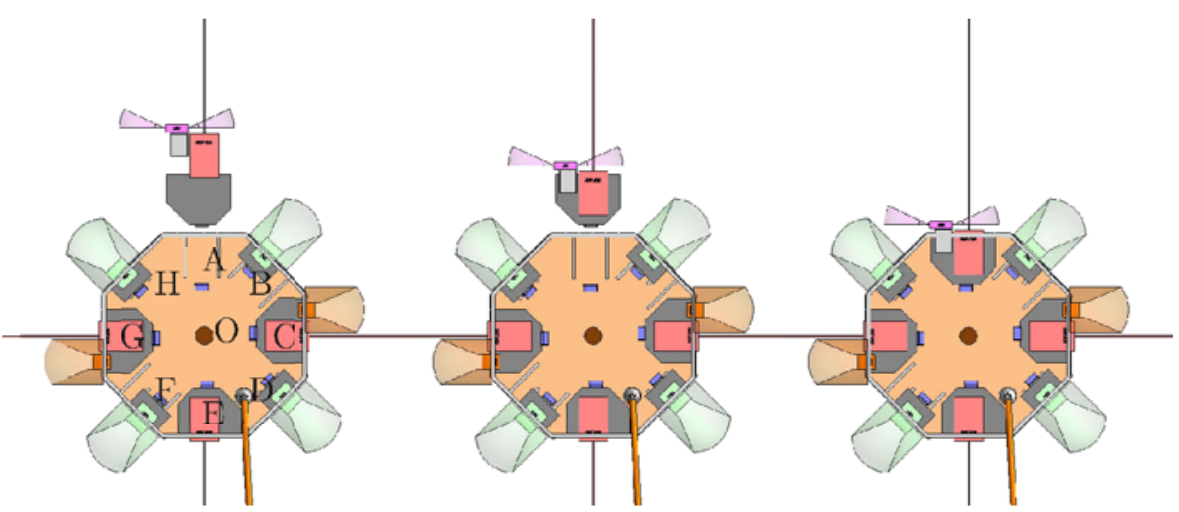

Fig. 5 Instruments mounted on universal plates [1]. For reasons of economy, Cross-Scale will utilise a single spacecraft bus design. Since the science payload is not identical for all spacecraft, a modular approach is adopted to accommodate different instruments in the same position. For ease of assembly, integration, and verification, a universal plate will be provided to instrument teams. Intrument mounting (left) and testing (middle) can be performed independently before the bays are then integrated, connected, and tested on the spacecraft (right). Plate locations used in Table 1 are lettered for convenience

overall turbulence spectrum, and specific fluctuations responsible for particle scattering and energisation.

AC search coil magnetometers extend waveform measurements up to $\sim 500, \mathrm{~Hz}$ and spectral information up to several kilohertz. These complete the characterisation of waves responsible for particle scattering and extend the turbulence analysis into the electron dissipation range.

Electric field antennae will be used to cover the range from DC to $100 \mathrm{kHz}$ on the inner spacecraft, where wave scattering and energisation of ions and electrons is a key priority for shocks and reconnection, and for the dissipation

Table 1 Cross-Scale payload by spacecraft

Instrument locations are given by lettered bays, and shown in Fig. 5; blanks indicate instruments not flown on that particular spacecraft; location $\mathrm{Z}$ refers to internal electronics

\begin{tabular}{|c|c|c|c|c|}
\hline \multirow[t]{3}{*}{ Instr } & \multicolumn{4}{|c|}{ Spacecraft/location on which deployed } \\
\hline & \multirow{2}{*}{$\begin{array}{l}\text { Electron } \\
\text { e } 1-2\end{array}$} & \multicolumn{2}{|l|}{ Ion } & \multirow{2}{*}{$\frac{\text { Fluid }}{\text { f 1-4 }}$} \\
\hline & & i 1 & i 2-4 & \\
\hline MAG & $\mathrm{F}$ & $\mathrm{F}$ & $\mathrm{F}$ & $\mathrm{F}$ \\
\hline $\mathrm{ACB}$ & B & B & B & B \\
\hline E2D & ACEG & $\mathrm{ACEG}$ & $\mathrm{ACEG}$ & \\
\hline E3D & $\mathrm{O}$ & & & \\
\hline EDEN & $\mathrm{Z}$ & $\mathrm{Z}$ & $\mathrm{Z}$ & \\
\hline ACDPU & $\mathrm{Z}$ & $\mathrm{Z}$ & $\mathrm{Z}$ & \\
\hline EESA & BDFH & & & \\
\hline CESA & & $\mathrm{BF}$ & BDFH & $\mathrm{D}$ \\
\hline ICA & & DH & & \\
\hline ECA & & & $\mathrm{AE}$ & \\
\hline HEP & & & $\mathrm{CG}$ & $\mathrm{H}$ \\
\hline ASP & $\mathrm{O}$ & & & \\
\hline CPP & $\mathrm{Z}$ & $\mathrm{Z}$ & $\mathrm{Z}$ & $\mathrm{Z}$ \\
\hline
\end{tabular}




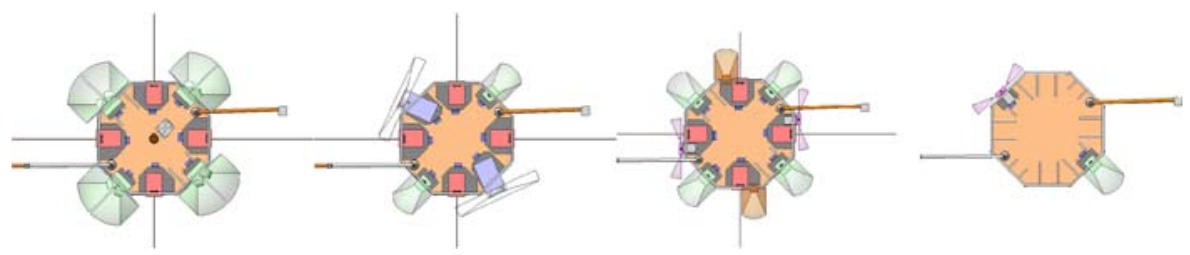

Fig. 6 Accommodation and fields of view of the scientific payload on the electron (left), ion (two middle), and fluid-scale (right) spacecraft [1]. Table 1 identifies the instruments and bay locations, which are lettered in Fig. 5

of turbulence. Electric measurements complement the magnetic ones to identify the electrostatic components of the waves and turbulence. The antennae will also be employed to measure the ambient DC electric field and the spacecraft floating potential. These, together with an electron density sounder, provide a high time resolution, high quality total electron density measurement that is central to the shock and reconnection science objectives, and that underpins the calibration of the particle instruments. Apart from the fluidscale spacecraft, all satellites will carry a crossed pair of wire boom antennae. At the electron scale all three components of the crucial reconnection electric field and the cross-shock electric fields will be completely characterised by the addition of rigid, dual-axial booms.

Electrostatic particle detectors of various designs will be carried on all spacecraft. These provide not only the basic plasma parameters (density, velocity, temperature, etc.) but also the full 3D velocity distribution functions. Collisionless plasma processes are driven by particle beams, temperature anisotropies, and other features that are not accessible to the low-order moments. Energy partition (heating and acceleration) amongst the various ion components requires some mass-resolving capability employing time-offlight techniques in concert with the basic electrostatic design that is utilised for electrons. The comprehensive instrument suite on the SCOPE mother spacecraft enables Cross-Scale to deploy a small number of core instruments to address the multi-scale aspects.

Modern analysers utilise a top-hat design which has a narrow field of view in one dimension. Full 3D velocity measurements require a full satellite spin period for a single sensor, which is adequate at the fluid scale. Multiple sensors, as either dual-heads within a single unit or multiple units, are deployed on the ion and electron scale spacecraft to increase the resolution and provide full 3D capability at the corresponding ion or electron time scales demanded by the science objectives. Additionally, deflector plates can be employed to increase the field of view for sub-spin resolution and reduce the number of sensors required. A combined electron/ion electrostatic analyser would greatly enhance the science return within payload mass restrictions by avoiding separate instruments for each species.

Energetic ion and electron detectors are required to investigate acceleration processes. The larger gyroradii and scales associated with these particles implies that not all spacecraft need to be so equipped, and the deployment 


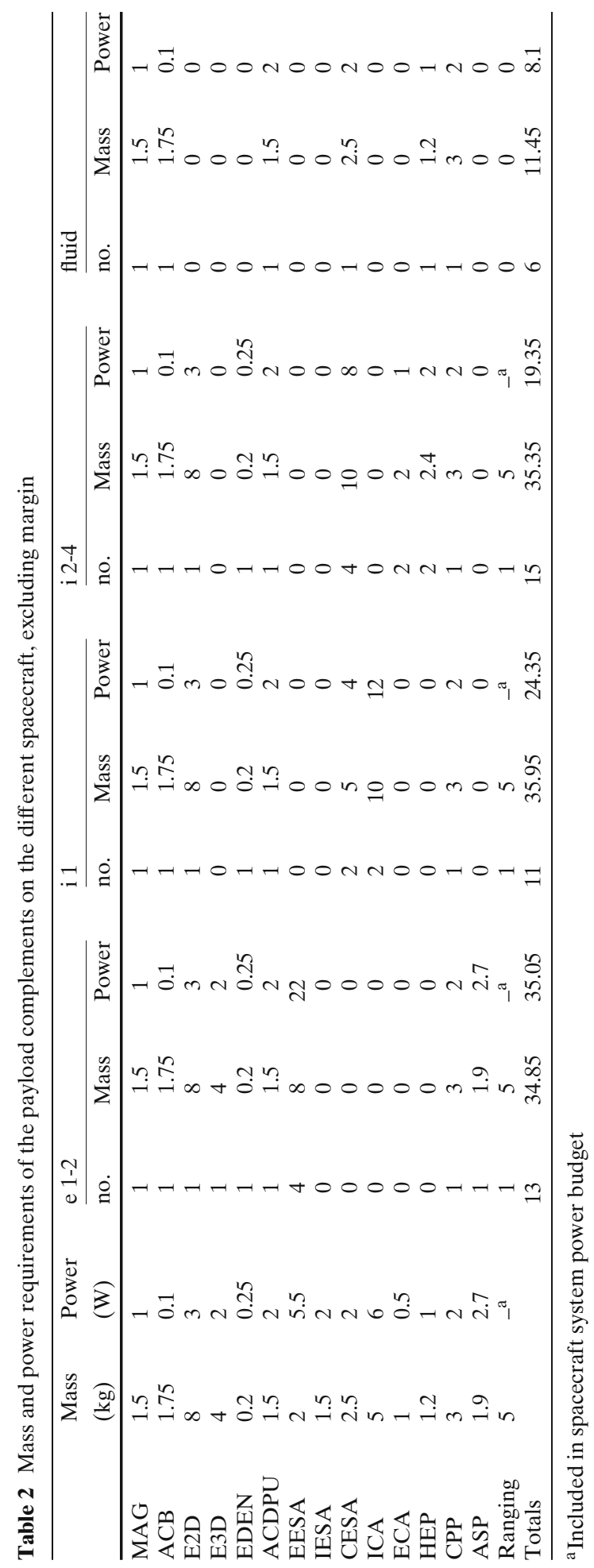




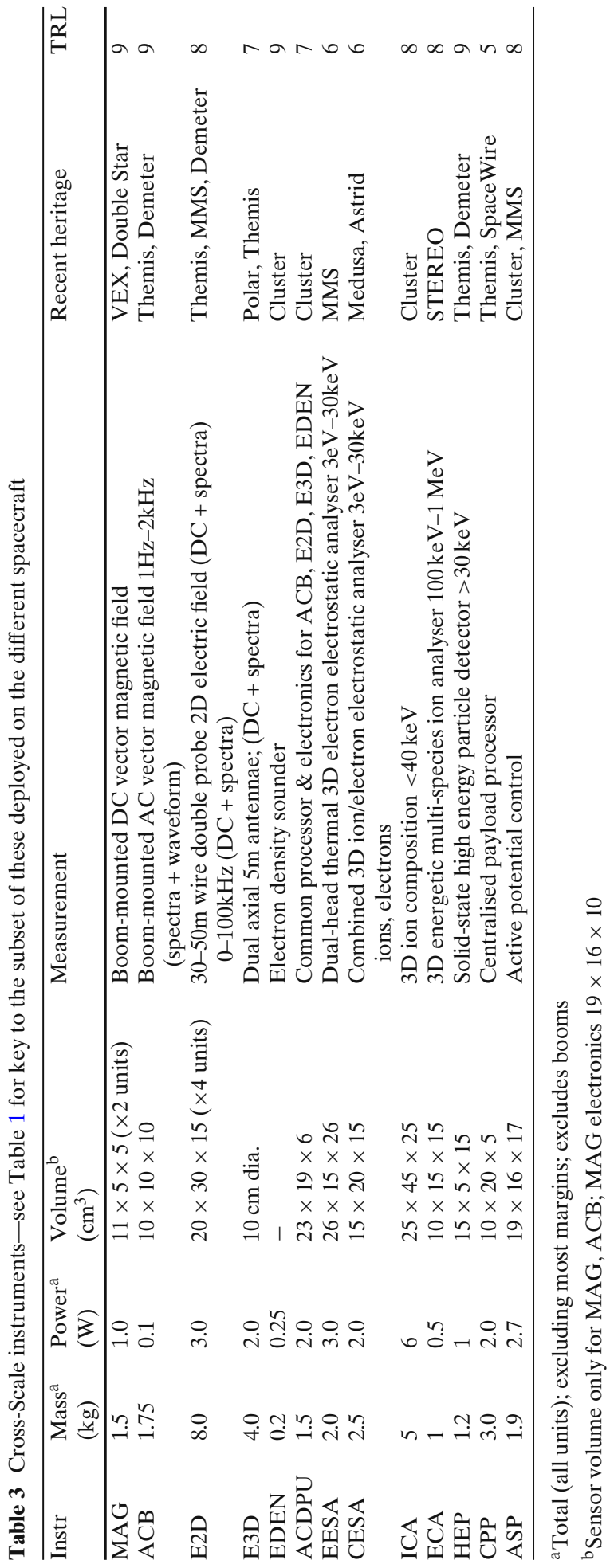


pattern shown in Table 1 represents a minimal configuration at each scale in a way which returns essential information on energetic ions, electrons, 3D ion velocity distributions, and ionic species.

Centralised onboard processing represents an attractive means of coordinating the payload, sharing (and hence reducing) resources. A dedicated wave processor has been successfully used in the past to coordinate common commanding, data taking, signal processing, and telemetry for electric and AC magnetic field instruments. A similar approach to particle instruments may be adopted. Alternatively, a distributed system taking advantage of modern communication and computing capabilities, such as SPACEWIRE, will be studied during the assessment phase. There are obvious and significant mass savings to be gained by reducing or eliminating one or several instrumentdedicated data processing units. This strategy needs to be developed in tandem with the multi-instrument bays in the current spacecraft design.

Active spacecraft potential control will be flown on the electron scale. This limits the contamination of the electron measurements by photoelectrons of spacecraft origin, and enables the low energy population to be resolved without large and sometimes uncertain corrections.

The basic characteristics of the instruments are described in Table 3. All instruments have recent flight heritage. Ongoing developments in mass, power, and science performance will be utilised to optimise the actual mission hardware.

\subsection{Data rates}

The science requirements met by the payload complement on the various spacecraft lead to a substantial data volume. That volume is estimated in Table 4, and used to allocate adequate spacecraft resources, telemetry, and ground station support.

The total mission data rate is $1.6 \mathrm{M}$-science words per second. A 16-bit science word (generous for the particle instruments) and a compression ratio of 10 (conservative given modern compression techniques for particle data, which dominates the data volume) would imply a mission data-rate of $2.6 \mathrm{Mbps}$ of which $31 \%$ can be accommodated in a nominal total downlink capability of $800 \mathrm{kbs}$. The actual downlink budget is driven by the subset of the orbit corresponding to the key target regions and boundary layers, so the full science objectives can be comfortably accommodated by this data rate. In fact, the majority of the data volume is generated by the electron scale spacecraft. Based on Table 4, these accumulate roughly 61 Gbytes of compressed data in two orbits (6 days). If adequate centralised onboard storage is not available for these spacecraft, a number of strategies exist to avoid compromising the science, including internal instrument memory, autonomous or predicted event triggers, higher compression, and down-sampling in energy-angle-time resolution. 


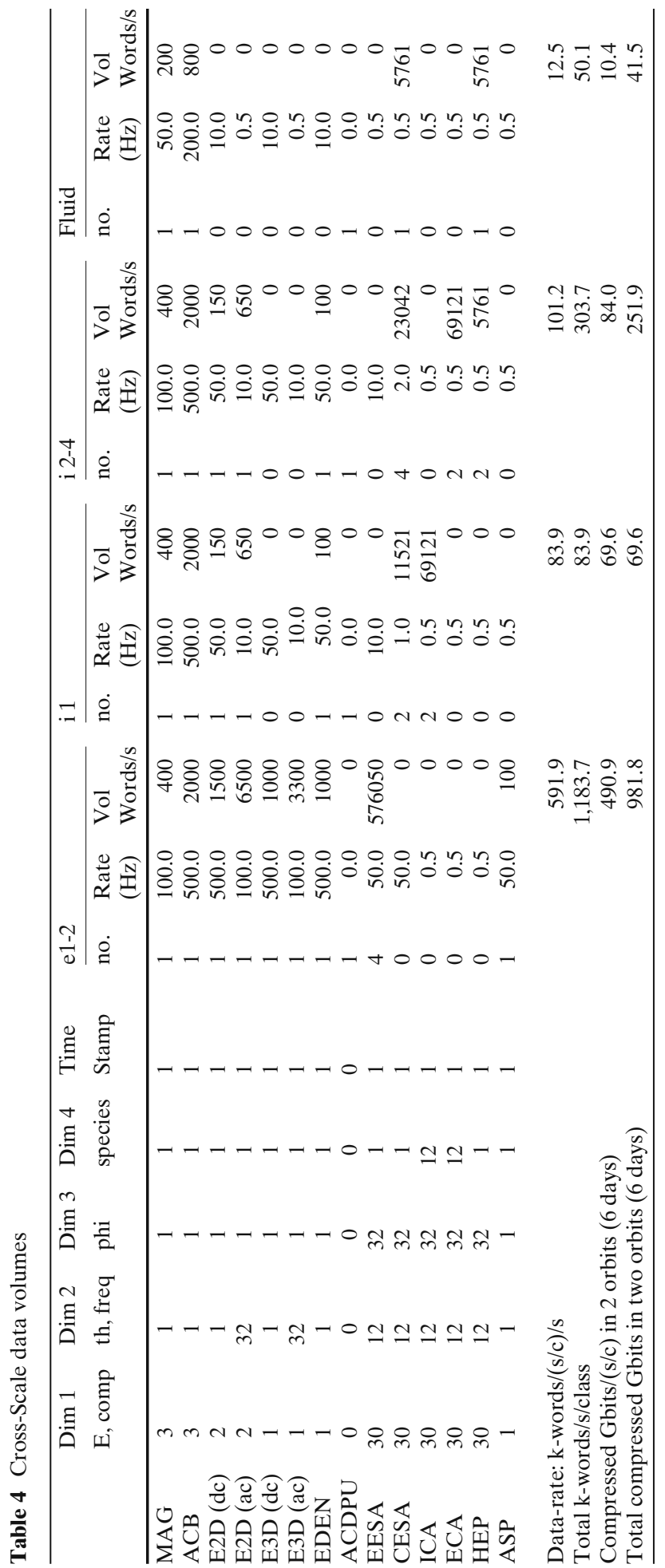




\subsection{Operations}

Limited instrument modes and minimal station-keeping are planned to restrict the ground segment resources required by the mission. A data segment is planned to ensure that the data are processed and calibrated, with highquality science data made available in a timely fashion for the widest possible community.

\section{Current status}

Cross-Scale has been selected by ESA to proceed to an Assessment Phase commencing late 2007. A Science Study Team will refine the mission and payload, which will be the subject of two parallel industrial studies that will be completed in 2009. Toward the end of 2009, ESA will make a further set of selections into a formal Definition Phase, from which two missions will be selected in 2011 for launch in 2017/2018.

\section{Conclusion}

Cross-Scale is an innovative next-generation mission with high-quality science objectives that attack fundamental physical phenomena in collisionless plasmas. It taps directly into European leadership in multi-point in situ space plasmas, and is built around flight-proven technologies. The mission has been selected by ESA to proceed to the Assessment Phase of Cosmic Vision missions. Further details and the mission proposal can be found on the community web-site at http://www.cross-scale.org.

Acknowledgements The EADS Astrium UK Ltd. study was funded by the Science and Technology Facilities Council of the UK. We wish to record here our appreciation to Marcel van den Berg for the tireless, thorough and good-humoured manner in which he liaised with utmost integrity between the community and the ESA-funded Technology Reference Study, and our deepest sadness at his untimely death.

\section{References}

1. EADS Astrium Ltd, UK: Cross-Scale: UK STFC-funded study. Internal report (2007)

2. Fujimoto, M., Tsuda, Y., Saito, Y., Shinohara, I., Kasaba, Y., Kojima, H.: The SCOPE mission. In: Favata, F., Sanz-Forcada, J., Giménez, A., Battrick, B. (eds.) ESA Special Publication, ESA Special Publication, vol. 588, 249 (2005)

3. Horbury, T., Louarn, P., Fujimoto, M., Baumjohann, W., Blomberg, L.G., Barabash, S., Canu, P., Glassmeier, K.H., Koskinen, H., Nakamura, R., Owen, C., Pulkkinen, T., Roux, A., Sauvaud, J.A., Schwartz, S.J., Svenes, K., Vaivads, A.: Cross-scale: a multi-spacecraft mission to study cross-scale coupling in space plasmas. In: Cluster and Double Star Symposium, ESA Special Publication, vol. 598 (2006)

4. Laval, J.P.: http://www.atmos.ucla.edu/ laval/Turbulence/turbulence.html (2007) 
5. Owen, C.J., Fazakerley, A.N., Schwartz, S.J., et al.: Multi-point, multi-scale investigations of fundamental plasma processes in the Earth's magnetosphere. In: Favata, F., Sanz-Forcada, J., Giménez, A., Battrick, B. (eds.) ESA Special Publication, ESA Special Publication, vol. 588, pp. 185 (2005)

6. Schwartz, S.J., Burgess, D.: Quasi-parallel shocks-a patchwork of three-dimensional structures. Geophys. Res. Lett. 18, 373-376 (1991)

7. Tanaka, K.G., Shinohara, I., Fujimoto, M.: Parameter dependence of quick magnetic reconnection triggering: a survey study using two-dimensional simulations. J. Geophys. Res. (Space Physics) 111(A10), 11 (2006). doi:10.1029/2006JA011968

8. The Cross-Scale Team: Cross-scale science priority document. http://www.cross-scale.org/ Documents/CrossScaleSciPriorityDocV1.3a.pdf (2007)

9. The Cross-Scale Team: Cross-scale: multi-scale coupling in space plasmas; response to ESA Cosmic Vision 2015-2025 call for proposals. http://www.cross-scale.org/Documents/Cross-ScaleCV-Proposal-28June2007.pdf (2007) 